\title{
Evaluación del impacto de un programa de formación en Entrevista Motivacional a enfermería nefrológica
}

\author{
Helena García-Llana ${ }^{1}$, Raquel Serrano², Hernán Cancio³ \\ ${ }^{1}$ Psicóloga Investigadora. $S^{\circ}$ de Nefrología. Hospital Universitario La Paz-IdiPAZ. Psicóloga Instituto Antae de \\ Psicología Aplicada y Counselling \\ ${ }^{2}$ Psicóloga Instituto Antae de Psicología Aplicada y Counselling \\ ${ }^{3}$ Director Instituto Antae de Psicología Aplicada y Counselling
}

\section{Resumen}

Introducción: Los profesionales de enfermería de cinco servicios de nefrología recibieron entrenamiento en Entrevista Motivacional mediante un taller de ocho horas centrado en como promover la adhesión al tratamiento en el paciente renal y su familia. El objetivo fue evaluar cambios en: competencia percibida en habilidades comunicativas especificas, respuesta empática espontanea e implantación de las habilidades in vivo medido a través de una rejilla observacional.

Material y Método: Diseño pretest-postest sin grupo control. La muestra estaba formada por 46 profesionales del ámbito de la enfermería nefrológica.

Resultados: Los resultados muestran cambios en la capacidad percibida de validar al paciente $(p<0,031)$, así como en autorregulación emocional $(p<0,014)$. La respuesta empática espontánea aumentó en los cuatro casos clínicos presentados, pero no llegó a alcanzar significancia estadística $(p>0,05)$. La rejilla observacional, implementada durante el entrenamiento, informó acerca de indicadores de éxito tales como: reducir el tono paternalista, mostrar calma, generar confianza y escuchar de manera activa.

Correspondencia:

Helena García-Llana

Servicio de Nefrología. Hospital Universitario La Paz-IdiPAZ

Paseo de La Castellana, 261.28046 Madrid

E-mail: helenagllana@hotmail.com
Conclusiones: Los resultados son consistentes con otros estudios que evalúan el impacto de los programas de entrenamiento en habilidades de comunicación en nefrología.

PALABRAS CLAVE: programa formativo; entrevista motivacional; adhesión al tratamiento; enfermería nefrológica.

\section{Abstract}

Introduction: Nursing professionals from five nephrology departments received training in Motivational Interviewing through an eight-hour workshop focused on how to promote adherence to treatment in renal patients and their families. The objective of the present study was to assess changes in: perceived competence in specific communication skills, spontaneous empathic response and readiness to apply in vivo communicative skills measured through an observation grid.

Material and Method: Pretest-posttest design without control group. The sample consisted of 46 nephrology nurses.

Results: The results show changes in the perceived competence to validate the patient $(p<0.031)$, as well 
as emotional self-regulation $(p<0.014)$. The empathic spontaneous response increased in the four clinical cases presented, but no statistical significance was reached $(p>0.05)$. The observation grid, implemented during the training, reported on indicators of success such as: reducing the paternalistic tone, showing calm, generating trust and active listening.

Conclusions: The results are consistent with other studies that evaluate the impact of communication training programs in nephrology.

KEYWORDS: training program; motivational interviewing; adherence to treatment; nephrology nursing.

\section{Introducción}

Los pacientes renales en tratamiento crónico sufren situaciones de difícil manejo, como la ansiedad ante el propio tratamiento renal sustitutivo, los déficit de información ante la polimedicación, el exceso de expectativas respecto al proceso de trasplante desembocando en momentos críticos en un paciente crónico. Los recursos personales de las enfermeras para afrontar y acompañar estas situaciones pueden facilitar la adhesión del paciente a los tratamientos y el éxito para afrontar la cronicidad ${ }^{1}$. A lo largo del proceso de la enfermedad, la motivación del paciente oscila debido a múltiples circunstancias. Además, se ve influenciada por la carga sintomatológica, cansancio emocional, disputas familiares, estrés laboral y/o problemas maritales influyendo sobre la motivación y el compromiso del paciente renal con su propio autocuidado. La atención individualizada en la consulta a través de un estilo de relación cercano y participativo ayudará a mantener y reforzar la motivación activa en momentos de riesgo o dificultad ${ }^{2}$. Los profesionales sanitarios que destacan en habilidades de comunicación han demostrado tener impacto en la confianza generada en los pacientes, la promoción de apoyo social, la autoeficacia y, por tanto, en la adhesión a los tratamientos 3. Harwood et al. describió como los programas formativos en el ámbito renal mejoran el trato paciente-personal sanitario, actuando como protector ante el estrés laboral asistencial ${ }^{4}$.

La comunicación terapéutica es una habilidad no innata y que es potencialmente moldeable mediante la formación y el aprendizaje. Por tanto, es necesario encontrar escenarios de aprendizaje en los que se cree un ambiente facilitador para detectar y reconducir cier- tos automatismos comunicativos de los que no siempre se es consciente y que pueden ser iatrogénicos en la relación con el paciente y su familia ${ }^{5}$. Por otro lado, cada vez nos encontramos más evidencia que reclama que se preste especial atención a los factores culturales a la hora de diseñar estos programas ${ }^{6}$.

La Entrevista Motivacional (EM) es una intervención directiva, altamente estructurada y centrada en el paciente que se focaliza en explorar y resolver la ambivalencia que acompaña todo cambio de conducta ${ }^{7}$. La EM busca activar el deseo de cambio y su efectividad. Ha sido probada en programas diseñados para pacientes renales avanzados ${ }^{8}$, siendo el paciente el que presenta las razones para cambiar y no el profesional. El profesional busca crear una atmósfera positiva para promover el autocuidado basándose en una serie de conceptos generales que están presentes en todo proceso de motivación para el cambio, articulando la gestión del cambio sobre el paciente ${ }^{9}$. La tarea del profesional es facilitar la expresión de la postura ambivalente y guiarle hacia una resolución que le conduzca al cambio. La persuasión directa, el enfrentamiento agresivo y la discusión no son métodos efectivos para resolver la ambivalencia. Es tentador intentar "ayudar" convenciendo al paciente de la urgencia del problema y de la necesidad del cambio, diciéndole lo que debe hacer, pero tales tácticas aumentan por lo general la resistencia del paciente y reducen la probabilidad de cambio.

En nuestro país existen trabajos previos del mismo grupo de investigación, que han demostrado un impacto positivo en programas formativos a la comunidad nefrológica ${ }^{10-13}$. Por tanto, nos planteamos el siguiente objetivo de estudio: Evaluar los cambios observados en el personal de enfermería en competencia percibida y respuesta empática espontanea, antes y después de la implantación de un taller monográfico de EM. Así mismo, en este estudio se aplica una rejilla observacional para describir la aplicación in vivo de determinadas habilidades durante el desarrollo del taller.

\section{Material y Método}

En este estudio se empleó un diseño cuasiexperimental pre-post sin grupo control. La muestra inicial está compuesta por un total de 46 profesionales de enfermería nefrológica de cinco hospitales españoles. 


\section{FORMACIÓN “PRACTICANDO CON LA ENTREVISTA MOTIVACIONAL EN ERC”}

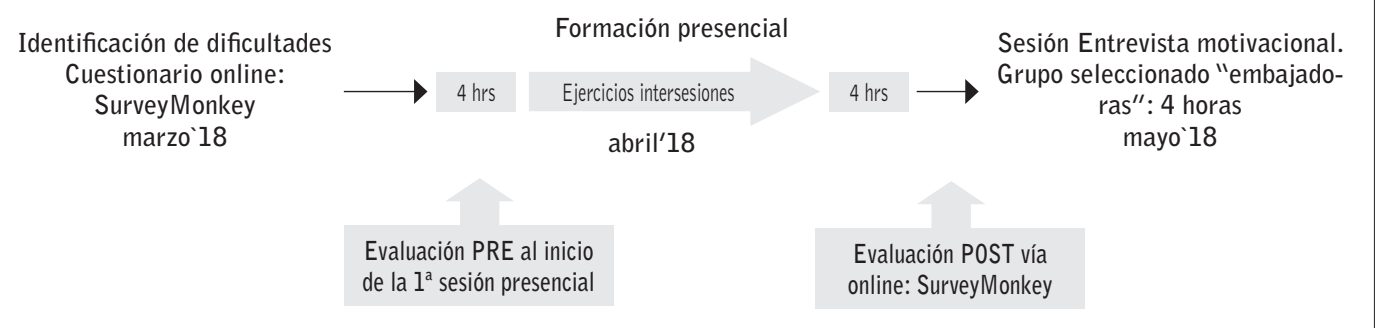

Figura 1. Diseño del programa de formación a enfermería nefrológica en EM. b) Competencia percibida en habilidades comunicativas específicas: consta de 6 ítems en una escala de respuesta tipo Likert que va desde el 1 (completamente incapaz) a 10 (completamente capaz).

c)Implicación en la relación enfermera-paciente: consta de un ítem en una escala de respuesta tipo Likert que va desde el 1 (distanciamiento) a 10 (absoluta implicación).

Los contenidos de los ítems han sido extraídos de la literatura ${ }^{14-15}$ y de estudios previos específicos por profesionales de nefrología ${ }^{13}$.

\section{Figura 1.}

El programa se inicia con una identificación de casos difíciles en la práctica diaria que se realiza on-line, y con la evaluación PRE. A continuación, se lleva a cabo la formación de 8 horas de manera intensiva en dos mañanas o tardes consecutivas. La evaluación POST también se lleva a cabo de manera on-line a los 15 días de recibir la formación. Además del taller de ocho horas, un grupo de diez "embajadoras de la EM", fue seleccionado, en base a su motivación y liderazgo dentro de los equipos, para asistir a una sesión de refuerzo de 4 horas. El objetivo de esta sesión fue entrenarse para desarrollar una sesión formativa en EM dentro de sus propios equipos para aumentar la difusión de las herramientas y la sostenibilidad de los cambios. Las embajadoras recibieron una presentación acerca de cómo orientar la preparación de dichas sesiones formativas.

\section{Instrumentos}

La evaluación del impacto del programa se llevó a cabo con un cuestionario elaborado ad-hoc en las siguientes áreas:

Cuestionario de Competencia Percibida: este cuestionario se estructura en tres áreas:

a) Eficacia percibida en el abordaje de la motivación al cambio en consulta: consta de un ítem en una escala de respuesta tipo Likert que va desde el 1 (completamente ineficaz) a 10 (completamente eficaz).
Adaptación de la herramienta de identificación de respuesta espontánea a nefrología (IRE) ${ }^{16-17}$. EI IRE consiste en la presentación breve de varias situaciones comunicativas difíciles que son susceptibles de causar sentimientos de malestar o ansiedad en el profesional. En esta versión, los respondientes deben elegir, de entre cuatro posibles alternativas, la respuesta que espontáneamente darían a cada situación. Las respuestas son mutuamente excluyentes y comprenden las siguientes categorías: 1 ) respuestas de estilo paternalista, 2) respuesta evasiva, 3 ) respuesta de estilo informativo, 4) respuesta motivacional. Aunque el IRE tiene originalmente 10 casos, en este estudio realizamos una adaptación (nefrología de adultos) de solamente cuatro casos para limitar el tiempo que los participantes pasaban completando las evaluaciones pre y post. En la Tabla 1 presentamos uno de los casos incluidos en el cuestionario IRE, con las respuestas incluidas en cada categoría.

\section{Rejilla observacional}

Se diseñó una rejilla observacional para ser utilizada durante los role-playings realizados en el taller. Uno de los participantes valoraba a un compañero en habilidades no verbales (mantiene el contacto ocular, postura adecuada, movimientos relajados etc.) y verbales (hace preguntas adecuadas, valida sentimientos, practica la escucha activa etc). Solo se tomó una medida post.

La evaluación del impacto se completó con la realización de una encuesta de satisfacción. 
Tabla 1. Ejemplo de situaciones y respuestas de la adaptación del cuestionario IRE empleado en este estudio.

CASO: Alberto tiene 27 años y acaban de trasplantarle por segunda vez. Ha
estado 7 años en hemodálisis después de perder su primer riñón por dejar de
tomar la medicación. En la consulta te dice: "Se que necesito bajar de peso
para cuidar mi riñón, ya perdi el otro porque no me tomé las pastillas, pero
es que me cuesta muchísimo ponerme serio con estos temas, a la mínima
oportunidad que tengo vuelvo a caer en mis antiguos hábitos

\section{Resultados}

\section{Características de la muestra}

Todos los profesionales que conformaron la muestra $(n=46,100 \%)$ respondieron al cuestionario de evaluación tanto en el pretest como en el postest. La muestra estaba formada por 41 mujeres ( $89 \%$ ) y en cuanto a la pertenencia del centro hospitalario, $11(23,9 \%)$ pertenecían al Hospital Universitario Miguel Servet de Zaragoza, $10(21,7 \%)$ al Hospital Universitario La Princesa de Madrid, $10(21,7 \%)$ al Hospital Sant Antoni Abad, $10(21,7 \%)$ al Vilanova i la Geltrú de Barcelona, 9 $(19,5 \%)$ al Hospital Universitario La Paz de Madrid y finalmente $9(19,5 \%)$ al Hospital Clinic de Barcelona.

La variables sociodemográficas recogidas no tuvieron relación con el impacto de adquisición de competencias. Resultados de la intervención

\section{Comparaciones de medias pre-post en competencia percibida}

Existen diferencias estadísticamente significativas en aplicación de la técnica de validación $(p=0,031)$ y en capacidad de autorregulación emocional del profesional

\section{Recogida de los datos}

Los datos se recogieron mediante los cuestionarios, realizados en cinco ediciones de un taller de 8 horas en tres comunidades autónomas (Madrid, Cataluña y Aragón) a lo largo de abril-mayo de 2018. El mismo fue impartido por dos psicólogas con experiencia en desarrollar formación en comunicación terapéutica a profesionales de nefrología.

\section{Análisis estadístico}

Para el análisis de los datos se empleó el paquete estadístico SPSS para Windows (versión 21.0). Para comparar la comparación pre-post en variables cualitativas se uso la $t$ de Student como prueba paramétrica y la $U$ de Mann-Whitney como prueba no paramétrica. Con respecto a las variables cualitativas se utilizó la prueba de Chi-cuadrado. Para estudiar la rejilla observacional se hizo un análisis resumen cualitativo entre tres investigadores expertos en impartir formación a profesionales sanitarios. Por último, se describen a través de frecuencias los resultados de la encuesta de satisfacción.
Tabla 2. Comparación de medias pre-post en las variables de competencia percibida.

\begin{tabular}{|c|c|c|c|}
\hline Variables & Media pre $(n=46)$ & Media post $(n=46)$ & $\mathbf{p}$ \\
\hline $\begin{array}{c}\text { ¿Cómo de eficaz te sientes en el } \\
\text { abordaje de la motivación al cambio } \\
\text { del paciente renal? }\end{array}$ & $6,74(\mathrm{DT}=1,49)$ & $7,29(\mathrm{DT}=1,57)$ & 0,234 \\
\hline $\begin{array}{l}\text { ¿Hasta qué punto te sientes capaz } \\
\text { de motivar al paciente renal en la } \\
\text { consulta para que realice cambios en } \\
\text { su estilo de vida? }\end{array}$ & $6,81(\mathrm{DT}=1,42)$ & $7,13(\mathrm{DT}=1,45)$ & 0,479 \\
\hline $\begin{array}{l}\text { ¿Hasta qué punto te sientes capaz de } \\
\text { implicar al paciente renal en su propio } \\
\text { autocuidado? }\end{array}$ & $7,15(\mathrm{DT}=1,41)$ & $7,50(\mathrm{DT}=1,28)$ & 0,273 \\
\hline $\begin{array}{c}\text { ¿Hasta qué punto te sientes capaz } \\
\text { de validar sin hacer sentir culpable } \\
\text { al paciente ante la no adherencia } \\
\text { terapéutica? }\end{array}$ & $6,73(\mathrm{DT}=1,45)$ & $8,00(\mathrm{DT}=0,93)$ & 0,031 \\
\hline $\begin{array}{l}\text { ¿Hasta qué punto te sientes capaz de } \\
\text { explorar las dificultades del paciente } \\
\text { renal para la adherencia terapéutica? }\end{array}$ & $6,92(\mathrm{DT}=1,28)$ & $7,79(\mathrm{DT}=1,31)$ & 0,097 \\
\hline $\begin{array}{l}\text { ¿Hasta qué punto te sientes capaz } \\
\text { de ayudar al paciente a afrontar las } \\
\text { situaciones difíciles de la enfermedad? }\end{array}$ & $6,94(\mathrm{DT}=1,32)$ & $7,50(\mathrm{DT}=1,14)$ & 0,157 \\
\hline $\begin{array}{l}\text { ¿Hasta qué punto te sientes capaz } \\
\text { de no irritarme ante la falta de } \\
\text { cumplimiento del paciente? }\end{array}$ & $6,90(\mathrm{DT}=1,93)$ & $8,00(\mathrm{DT}=1,25)$ & 0,014 \\
\hline $\begin{array}{l}\text { ¿Cuál suele ser tu postura o reacción } \\
\text { más frecuente en la comunicación con } \\
\text { los pacientes renales y sus familias? }\end{array}$ & $6,88(\mathrm{DT}=1,63)$ & $7,54(\mathrm{DT}=1,47)$ & 0,248 \\
\hline
\end{tabular}


Tabla 3. Casos clínicos: comparación de aciertos en la respuesta motivacional pre-post de la herramienta IRE.

\begin{tabular}{|lllc|}
\hline CASOS CLÍNICOS IRE & $\begin{array}{l}\text { Porcentaje pre respuesta } \\
\text { motivacional (DT) }\end{array}$ & $\begin{array}{l}\text { Porcentaje post respuesta } \\
\text { motivacional (DT) }\end{array}$ & p \\
\hline Caso 1 & 56,3 & 95,8 & n.s \\
\hline Caso 2 & 47,9 & 86,3 & n.s \\
\hline Caso 3 & 47,9 & 75,0 & n.s \\
\hline Caso 4 & 52,1 & 79,2 & n.s \\
\hline
\end{tabular}

satisfacción nos muestran que los participantes han valorado todas las categorías exploradas por encima de 9 sobre 10 (Ver Figuras 2 y 3 ). El $100 \%$ de los participantes refieren que el curso les ha aportado alguna herramienta para despertar la motivación en el paciente. De la misma manera, el $100 \%$ de los participantes consideran hacer algún cambio en la forma de abordar al paciente a consecuencia de $(p=0,014)$, en la dirección de ser superiores una vez realizado el curso. El resto de los ítems aumentaron su media en la dirección esperada sin alcanzar significancia estadística. Ver Tabla 2.

\section{Comparaciones de medias pre-post en la herramien- ta IRE}

La respuesta empática aumentó de manera significativa en los cinco casos clínicos presentados, pero sin alcanzar significancia estadística $(p>0,05)$. Ver Tabla 3.

\section{Aplicación de la rejilla observacional}

Se han recogido y procesado 19 rejillas de observación. Todas las observadoras registraron habilidades de comunicación verbal y no verbal durante los role-play implementados en el curso. Las cinco categorías más destacables han sido: "reducción del tono paternalista", "movimientos relajados", "genera confianza" y "practica la escucha activa".

\section{Encuesta de satisfacción}

El $100 \%$ de la muestra respondió al protocolo de evaluación completo. Los resultados de la encuesta de haber asistido al curso. Algunos de los cambios descritos son: "ser más tranquila al plantear los temas y posibles soluciones", "no enjuiciarle", "Hacer más hincapié en la validación para poder explorar miedos y ayudarles en la adhesión terapéutica", etc.

\section{Discusión}

Los principales hallazgos de este estudio son que la participación de las enfermeras nefrológicas en un programa formativo en EM mejoró la capacidad percibida en aplicar la técnica de validación, así como en la autorregulación emocional. Ambas herramientas vienen demostrando su efectividad en el ámbito de la comunicación terapéutica dentro del contexto del Counselling ${ }^{18-19}$.

La respuesta motivacional aumentó en los cuatro casos clínicos, pero sin alcanzar significancia estadística, seguramente debido al escaso tamaño muestral. Por otro lado, existe evidencia de que se mostraron habilidades in vivo de comunicación terapéutica (verbal y no verbal) durante los role play. Autores referentes en esta área,

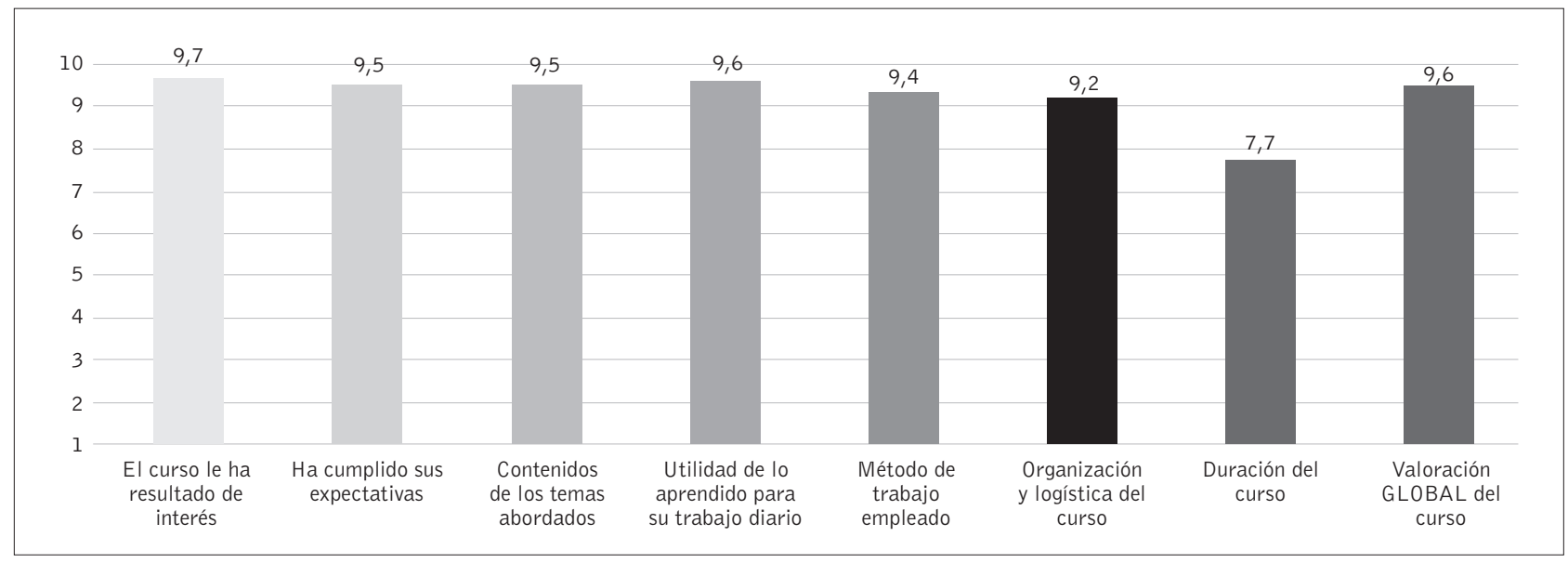

Figura 2. Satisfacción con el programa formativo en EM. 


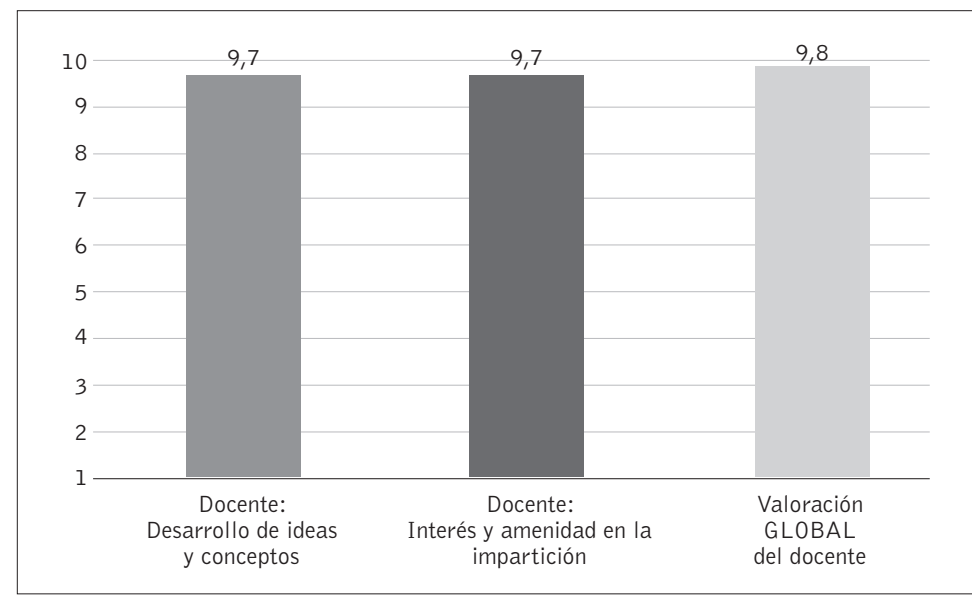

Figura 3. Satisfacción con el equipo docente responsable de la formación. el encontrar relaciones entre las mismas y la adquisición de competencias. Futuros estudios deben de incorporar mayor número de variables en la recogida de datos, así como ser dotados con más recursos para poder evaluar el impacto mediante entrevistas a pacientes 0 bien mediante observadores en consulta ${ }^{21}$.

Por tanto, concluimos que tenemos la responsabilidad de seguir explorando diferentes formatos de entrenamiento, así como el uso de instrumentos de evaluación sencillos y eficaces, que puedan aplicarse a diferentes escenarios dentro del ámbito sanitario. En el contexto de la atención al paciente renal y su familia, el conocimiento de la tecnología médico-biológica sirve de poco si no se contemplan los factores facilitadores del auto-cuidado como son el soporte emocional y la promoción de la motivación al cambio, todo ello sin olvidar la atención a los aspectos emocionales de los propios profesionales.

\section{Conflicto de interés}

Este estudio ha estado impulsado y financiado por Vifor Fresenius Medical Care Renal Pharma, que ha participado en este estudio en el diseño y en la implementación de los aspectos logísticos juntamente con el equipo coordinador. La autora Dra H García-Llana, declaran tener conflicto de interés habiendo recibido honorarios como consultores expertos en advisory board del estudio. Todos los autores han participado en la revisión del manuscrito. El diseño, la recogida de datos, análisis estadístico y elaboración del manuscrito fueron realizados por Instituto ANTAE de Psicología Aplicada y Counseling Madrid, España).

\section{Agradecimientos}

A Vifor Fresenius Medical Care Renal Pharma España S. L. por dar apoyo a esta iniciativa a través de su Programa NEFRALIA ${ }^{\circledR}$ de apoyo al paciente renal y su familia.

Recibido: 5 noviembre 2018

Revisado: 8 noviembreo 2018

Modificado: 19 noviembre 2018

Aceptado: 23 noviembre 2018 pleados. Por otro lado, las variables sociodemográficas recogidas de la muestra fueron limitadas y eso dificultó 


\section{Bibliografía}

1. Mármol-López MA, Miguel-Montoya I, Montejano-Lozoya R, Escribano-Perez A, Gea-Caballero V, Hontangas-Ruiz $A$. Impacto de las intervenciones enfermeras en la atención a la cronicidad: Revisión sistemática. Rev Esp Salud Pública. 2018;92(27): 1-15.

2. McCarley P. Patient empowerment and motivational interviewing: engaging patients to self-manage their own care. Nephrology Nursing. 2010; 36(4):409-13.

3. Street Jr RL, Makoul G, Arora KJ y Epstein RM. How does communication heal?: Patahaways linking Clinician-patient communication to health outcomes. PEC. 2009;74:295-301.

4. Harwood L, Ridley J, Wilson B, Laschinger HK. Workplace empowermentand burnout in Canadian nephrology nurses. The CANNT Journal. 2010;20 (2):12-8.

5. Kruijver IP, Kerkstra A, Francke AL, Bensing JM y Van de Wiel HB. Evaluation of communication training programs in nursing care: a review of the literature. PEC. 2000;39:129-45.

6. Pun KJH, Chan EA, Wang S y Slade D. Health professional-patient communication practices in East Asia: An integrative review of an emerging field of research and practice in Hong Kong, South Korea, Japan, Taiwan, and Mainland China. PEC. 2018; 101(7):1193-206.

7. Miller W, Rollnick S. La entrevista motivacional. España: Paidos. 2015.

8. García-Llana H, Remor E, del Peso G, Celadilla 0 , Selgas R. Motivational Interviewing promotes adherence and improves wellbeing in pre-dyalisis patients with Advanced Chronic Kidney Disease. J Clin Psychol Med Settings. 2014;21:103-15.

9. Treasure J. Motivational interviewing. Advances in Psychiatric Treatment. [Internet] 2004. [Consultado 18 Agosto 2018]; 10(5):331-7. Disponible en: http://www.motivationalinterviewing.org/

10. García-Llana H, Barbero J, Remor E, Díaz-Sayas $L$, Rodríguez-Rey $R$, del Peso $G$ et al. Impacto de un curso interdisciplinar de Counselling y apoyo en la toma de decisiones a profesionales de un servicio de nefrología. Nefrología. 2011;31(3):322-30.

11. García-Llana H, Barbero J, Remor E, Celadilla Díez 0, Trocoli Gonzalez F, del Peso Gilsanz G et al. Beneficio de la realización de un taller en gestión emocional para enfermería nefrológica. Enferm. Nefrol. Revista Española de la Sociedad de Enfermería Nefrológica. 2012;15 (3):176-81.

12. García-Llana H, Rodríguez-Rey R, Selgas R. Formación en asesoramiento psicológico (Counselling) y apoyo emocional a residentes de Nefrología: Estudio Piloto. Salud i Ciencia. 2014;20:362-7.

13. García-Llana H, Bajo MA, Barbero J, del Peso G y Selgas R. The Communication and Bioethical Training (CoBiT) Program for assisting dialysis decision-making in Spanish ACKD Units. Psychology, Health \& Medicine. 2017;22(4):474-82.

14. Carvalho IP, Pais VG, Almeida SS, Ribeiro-Silva $R$, Figuereido-Braga $M$, Teles $A$. et al. Learning clinical communication skills: Outcomes of a program for professional practicioners. PEC. 2011;84:84-9.

15. Smith RC, Mettler JA, Stoffelmayr BE, Lyles JS, Marshall $A A$, van Egeren $L F$, et al. Improving residents' confidence in using psychosocial skills. J Gen Intern Med. 1995;10:315-20.

16. Bermejo JC, Carabias R, Villacieros M, Belda RM. Efecto de un curso relacional sobre la elección de respuesta espontánea e identificación de respuesta empática en alumnos de Medicina. Medipal. 2010; 17(5):262-8.

17. Bermejo JC, Carabias R, Villacieros M. Efecto de un curso de relación de ayuda sobre la elección de respuesta espontánea e identificación de respuesta empática en alumnos de enfermería. Gerokomos. 2012;23(1):23-8.

18. Benito $E$, Arranz P, Cancio H. Herramientas para el autocuidado del profesional que atiende a personas que sufren. FMC. 2011;18(2):59-65.

19. Costa M, Arranz P. Comunicación y Counselling en Psicooncologia. El encuentro con el paciente oncológico. En: Cruzado Rodríguez JA, coord. Manual de Psicooncología. Tratamientos psicológicos en 
pacientes con cáncer. Madrid: Pirámide; 2013. P. 55-78.

20. Lane $C$, Hood K y Rollnick S. Teaching motivational interviewing: Using role play is as effective as simulated patients. Mededuc. 2008;42:637-44.

21. Roter D, Larsson S. The Roter Interaction Analysis System (RIAS): Utility and flexibility for analysis of medica interaction. PEC. 2002;46:243-51.

22. Lundahl B, Moleni T, Burke BL, Rutters R, Tollefson D, Butler C, Rollnick S. Motivational Interviewing in medical care settings: A systematic review and meta-analysis of randomized control trials. PEC. 2013;93:157-68.

Este artículo se distribuye bajo una Licencia Creative Commons Atribución-NoComercial 4.0 Internacional. https://creativecommons.org/licenses/by-nc/4.0/

\section{Open Access (c) (1) (}

